\title{
Les Chouans de Balzac: défaite des sujets et production de l'avenir
}

\section{Chia-ping Kan}

\section{(2) OpenEdition}

1 Journals

\section{Édition électronique}

URL : https://journals.openedition.org/studifrancesi/2943

DOI : 10.4000/studifrancesi.2943

ISSN : 2421-5856

Éditeur

Rosenberg \& Sellier

\section{Édition imprimée}

Date de publication : 1 juillet 2013

Pagination : 246-261

ISSN : 0039-2944

\section{Référence électronique}

Chia-ping Kan, «Les Chouans de Balzac: défaite des sujets et production de l'avenir », Studi Francesi

[En ligne], 170 (LVII | II) | 2013, mis en ligne le 30 novembre 2015, consulté le 31 janvier 2023. URL: http://journals.openedition.org/studifrancesi/2943; DOI : https://doi.org/10.4000/studifrancesi.2943

\section{(c) (i) $\odot$}

Creative Commons - Attribution - Pas d'Utilisation Commerciale - Pas de Modification 4.0 International - CC BY-NC-ND 4.0

https://creativecommons.org/licenses/by-nc-nd/4.0/ 


\title{
Les Chouans de Balzac: défaite des sujets et production de l'avenir
}

\begin{abstract}
In Le dernier Chouan ou la Bretagne en 1800 (1829) Balzac proposes an analysis of French political situation by connecting three characters: Marie de Verneuil, the Marquis de Montauran and the commander Hulot. The identity of Marie, illegitimate child of the Duke de Verneuil and secret agent of the Revolution, is related to the complexity of the relations between some political systems which are opposite and complementary at the same time. In the end, the death of the two protagonists after their marriage is meaningful: it represents the fusion between two incompatible systems. Montauran and Hulot have been as "loyal enemies." After the death of the couple-enemies, and at the price of their sacrifice, the revolutionary military becomes a marshal and an executor of the aristocrat.
\end{abstract}

In Le dernier Chouan ou la Bretagne en 1800 (1829) Balzac propone un'analisi della situazione politica francese facendo interagire tre personaggi: Marie de Verneuil, il Marchese di Montauran e il comandante Hulot. L'identità di Maria, figlia illegittima del Duca di Verneuil e agente segreto della Rivoluzione, è in relazione con la complessità dei rapporti tra certi sistemi politici che sono al tempo stesso opposti e complementari. Alla fine, la morte dei due protagonisti dopo il loro matrimonio è significativa: rappresenta la fusione tra due sistemi incompatibili. Montauran e Hulot sono stati come "nemici leali". Dopo la morte della coppia-nemici, e a prezzo del loro sacrificio, il militare rivoluzionario diventa maresciallo e giustiziere degli aristocratici.

Le but de ce travail est d'analyser la position politique de Balzac telle que sa création romanesque permet de l'appréhender. Parmi tous les points d'accès à cette compréhension, la première œuvre signée par Balzac ${ }^{1}$, après l'échec de son entreprise d'imprimerie en août 1828, mérite que nous lui accordions toute notre attention: Le Dernier Chouan ou la Bretagne en 1800, dont la date de publication (1829) confirme la position de point de départ de Balzac par rapport à ses principaux romans. Cette publication témoigne ainsi de la volonté de Balzac de distinguer ce chef-d'œuvre de ses œuvres de jeunesse, rejetées comme «littérature marchande» et signées de pseudonymes. C'est donc, en quelque sorte, la première œuvre de la vraie aventure balzacienne.

La primauté que Balzac, jeune écrivain encore, décide d'attribuer à ce roman le rend encore plus important, notamment après tant d'années de réflexions ${ }^{2}$; dans l'«Avertissement du Gars»33, Balzac dénonce «cette prostitution de la pensée qu'on nomme: la publication» ${ }^{4}$. Mais ici, cette publication est considérée comme le premier succès; ce roman peut être vu comme une sorte de «préface» de La Comédie bumaine.

(1) La Préface de l'édition Furne commence par: «Cet ouvrage est mon premier, et lent fut son succès [...]», CH. (La Comédie bumaine), sous la direction de Pierre-Georges CAstex, Paris, Gallimard, «La Pléiade», 12 voll., 1976-1981, t. VIII, p. 903.
(2) Voir N. Mozet, Balzac au pluriel, PUF, Paris, 1990, début de l'ouvrage.

(3) Premier titre du roman.

(4) Les Chouans, CH., cit., t. VIII, p. 1669. 


\section{Le pivot du roman et de l'Histoire: Marie de Verneuil}

Le choix des personnages du Dernier Chouan ou la Bretagne en 1800 est très intéressant. Le marquis de Montauran et le général Hulot représentent deux conceptions politiques clairement opposées: l'Ancien Régime et la République; tandis que Marie de Verneuil, fille «naturelle» ${ }^{5}$ du duc de Verneuil, occupe une place indécise dans les combats acharnés entre chouans et républicains. Toute l'œuvre tourne autour de ce sujet: à quel camp appartient-elle? Officiellement, elle est envoyée par le ministre de la police, Fouché, pour séduire un «ennemi», Alphonse de Montauran, et le livrer à la République. Néanmoins, son attitude incertaine rend les choses beaucoup plus compliquées.

Dans les descriptions que fait le narrateur de ce personnage, les choses ne s'arrangent pas. À plusieurs reprises, l'éducation et les comportements spécifiquement nobles de Marie de Verneuil sont mis en évidence. Cette fille «spirituelle», d'une beauté extraordinaire, maitrise parfaitement l'art de s'exprimer et les coutumes de la Cour. Même pour un vieux courtisan comme le comte de Bauvan ${ }^{6}$, elle dégage une «noblesse» et une «fierté» «imposantes» ${ }^{7}$, qui le «désarme[nt]», puis, le «sédui[sent]»: «Ce pauvre comte se laissa prendre à la manière innocente dont sa libératrice avait accepté deux ou trois compliments assez bien tournés»?. Ce gentilhomme ainsi dompté lui sera fidèle jusqu'à la fin. C'est donc une jeune femme dont les «manières» sont incontestablement «nobles». Des «manières» qui sont en désaccord permanent avec sa mission républicaine.

D'ailleurs, sa «passion» brûlante pour le marquis de Montauran n'est-elle pas aussi un indice de sa «noblesse»? Les calomnies de Mme du Gua, et l'influence qu'elle a sur le marquis n'arrivent pas à détruire l'amour que Marie éprouve pour Montauran, et qui est devenu sa principale raison d'existence. Ainsi, lorsque Marie a l'impression d'être trahie par Montauran, l'idée de «se venger» devient «[sa] seule pensée, [son] unique passion $\gg^{10}$. Ce sont donc des sentiments complexes et entiers en même temps; elle aime et sa passion la détruira. Cette grandeur ne laisse pas de doute sur ses sentiments nobles: «j'ai compris hier qu'on vécût pour aimer, et je comprends aujourd'hui qu'on puisse mourir pour se venger» ${ }^{11}$.

À l'origine, certes, c'est grâce à la promesse d'une belle récompense qu'elle accepte sa mission. Néanmoins, une fois qu'elle a conscience de ses sentiments pour Montauran, l'argent n'a plus de sens pour elle. Â côté des autres personnages, les nobles, les chouans ou les républicains qui «dépouillent» les cadavres, qui ne se battent que par intérêt, et ne respectent que l'argent, les sentiments de Marie n'ont pas de prix. Même dans l'amour sincère entre Francine et Marche-à-terre, l'argent que «leur bienfaitrice» ${ }^{12}$, Marie, donne à Francine joue un rôle décisif, jusqu'à retourner complètement la situation. Grâce à cette générosité ${ }^{13}$ envers Francine, Marie est mira-

(5) Op. cit., p. 1143 (p. 286 dans l'édition de 1829, établie par Andrew Olivier; toutes les pages qui renvoient à l'édition d'Andrew Olivier seront mises entre parenthèse). Rappelons que l'édition de 1829 du Dernier Chouan ou la Bretagne en 1800 a été l'objet de deux importantes corrections de l'auteur: en 1834 (Les Chouans ou la Bretagne en 1799) et en 1845 (le volume XIII de La Comédie bumaine chez Furne). Cependant, nous pensons que les corrections n'ont pas changé fondamentalement le sens de l'œuvre. La première édition a été publiée telle quelle par A. OLIVIER, Editions de l'originale, coll. Les romans de Balzac, Toronto, 2005.
(6) Ce personnage était anonyme dans la version de 1829: «le comte de ***»».

(7) Op. cit., p. 1106 (p. 241)

(8) Op. cit., p. 1107 (p. 243).

(9) Op. cit., p. 1106 (p. 242).

(10) Op. cit., p. 1064 (p. 191).

(11) Ibid. $1^{\text {rer }}$ éd.: «on vit pour l'amour, mais on meurt pour se venger» (p. 192).

(12) Op. cit., p. 1042 (p. 165).

(13) «Cette belle demoiselle [...] a eu soin de moi. [...] J'ai maintenant deux cents livres de bonnes rentes. Enfin mademoiselle m'a acheté pour cinq cents écus la grande maison à mon oncle Tho- 
culeusement sauvée des griffes de Pille-Miche, et mise en sécurité par Marche-à-terre, qui est normalement un représentant intransigeant des chouans.

En effet, tout au long du roman, il n'y a que Marie, son amant et Hulot qui restent indifférents à l'argent. Marie refuse dignement la proposition des «diamants», des «bijoux», des «équipages», de l'«or» de d'Orgement de Fougères: «l'argent n'est rien pour moi» ${ }^{14}$. Le «dépouillage» des morts n'intéresse ni Montauran ni Hulot: «Soldats, s'écria Hulot d'une voix sévère, je vous défends de partager ces haillons. Formez vos rangs, et plus vite que ça!» ${ }^{15}$. Même si Mme du Gua soupçonne que le marquis n'accepte sa dangereuse mission que «pour mériter la main de Mlle d'Uxelles» ${ }^{16}$, l'attitude de Montauran démentira bientôt cette interprétation. La préoccupation de ces trois personnages va donc bien au-delà. Ils n'ont chacun qu'une «conviction»: pour Marie, il s'agit d'amour; pour Montauran et Hulot, il leur faut remporter la victoire, pour le Roi ou pour la République.

Idéologiquement toutefois, Marie se situe dans le camp républicain. Avant de s'associer avec le policier Corentin, d'obtenir la signature du ministre Fouché pour commencer sa mission et d'être ainsi «protégée par les troupes du gouvernement» ${ }^{17}$, Mlle de Verneuil était même Mme Danton ${ }^{18}$. Même si ce mariage n'a duré que quelques jours, et qu'elle n'a aucune estime ni pour le parti qu'elle représente ni pour la personne qui l'engage, et que la motivation de cet engagement n'a rien à voir avec les raisons politiques, Marie est une femme «républicaine». Voici ce qu'elle explique à Francine, sa «servante» ${ }^{19}$ et amie intime:

Je hais cette entreprise encore plus que celui [Corentin] dont la langue dorée me l'a expliquée $[\ldots]$ je ne me serais pas rendue à leurs désirs, si je n'avais pas entrevu dans cette ignoble farce un mélange de terreur et d'amour qui m'a tentée ${ }^{20}$.

Malgré la franchise de ce discours, Francine n'arrive toujours pas à bien saisir les rapports entre sa maîtresse et Corentin, qui surveille Marie de près. Cette dernière prétend qu'elle ne supporte pas la «répugnante ${ }^{21}$ présence de l'espion, mais, en même temps, elle accepte son aide et ses conseils. Après le massacre qui a été perpétré au château de la Vivetière, Marie se met sous la protection de Corentin: elle accepte sa présence auprès d'elle et sa proposition de louer pour elle une maison à Fougère. De plus, c'est justement sous l'influence de Corentin que Marie a été engagée dans sa mission. Ainsi, son attitude face à ce policier amoureux d'elle rend la situation complexe.

Cette énigme persiste et la situation ne s'éclaircit que dans la dernière partie du roman, où on apprend enfin l'identité de Mlle de Verneuil. C'est bien une femme noble, mais à moitié. Cet élément, souligné comme essentiel, au moins pour le mar-

mas, et j'ai deux mille livres d'économies», op. cit., p. 998 (p. 120).

(14) Op. cit., p. 1088. 1ère éd.: «l'argent n’est pas le but de ma course!» (p. 219).

(15) Op. cit., p. 1170 (p. 316)

(16) Op. cit., p. 1135 . 1ère éd.: «pour mériter la main de mademoiselle de R...n» (p. 277).

(17) Op. cit., p. 968 (p. 84).

(18) Op. cit., p. 1145 (p. 288). Voir la note de la page 1811 de l'édition de la Pléiade. Dans les manuscrits et les premières éditions de Balzac, Marie est «liée» à Danton. Ce n'est qu'à partir de l'édition Vimont, 1834, qu'elle est «mariée» à Danton. Dans tous les cas, la volonté de Balzac de «lier» Marie de Verneuil à Danton est claire.
(19) D'après Mme du Gua, le vocabulaire douteux employé par Marie prouve son identité républicaine: «Oh! temps d'orage, dévouement, madame, et la servante! dit-elle, ce ne doit pas être Mlle de Verneuil, mais une fille envoyée par Fouché», (op. cit., p. 982). Néanmoins, le mot «servante» n'est pas dans la version de 1829 et l'identité que se donne Marie avait encore de la crédibilité aux yeux de Mme de Gua: «Oh! - temps d'orage - dévouement, Madame... c'est peut-être mademoiselle de Verneuil! - mais pourquoi n'a-telle pas de poudre?» (p. 101).

(20) Op. cit., p. 969 (p. 86).

(21) Op. cit., p. 1155 (p. 190). 
quis et pour son entourage, est bien la clé du roman: «Êtes-vous une jeune femme belle, NOBLE et spirituelle? [...] $\gg^{22}$, telle est la question posée par le marquis de Montauran à Marie de Verneuil. La réponse ne sera donnée que bien plus tard, lorsque Marie racontera son origine et ses aventures.

L'identité bâtarde de Marie n'est donc pas un hasard; au contraire, elle est le reflet du rapport entre deux systèmes politiques. Cette jeune et belle femme porte un nom noble mais travaille pour la République; elle doit livrer Montauran mais est tombée éperdument amoureuse de lui, et elle est prête à tout lui sacrifier. Tout est contradictoire chez elle. Sa position est difficile à définir car elle n'en a aucune qui soit stable. Elle réagit suivant les circonstances, guidée par l'amour et ses sentiments, sans réel calcul politique. En trahissant d'abord le Roi, puis la République, Marie prétend qu'elle n'a besoin que d'être aimée par Montauran pour se sentir au «paradis», tout en sachant que cet amour ne lui apportera que la «mort»:

Oh! il m'aime ou il ne m'aime pas! répondit-elle, deux mots qui pour moi sont le paradis ou l'enfer. Entre ces deux extrêmes, je ne trouve pas une place où je puisse poser mon pied ${ }^{23}$.

Car son amour est impossible comme le rappelle Corentin à Galope-Chopine: «On ne peut pas être longtemps à la fois l'homme des Chouans et l'homme des Bleus $\gg^{24}$.

Malgré tout, soutenue par la puissance de l'amour, Marie est prête à s'affranchir, une fois de plus, de sa position politique. Cette femme républicaine en arrive à reconnaître la grandeur de l'action de son amant royaliste, qui se montre «si supérieur» ${ }^{25}$, qui se bat pour le roi, incarnation de la France. Cette vision du chouan, purement royaliste, arrive ainsi à trouver une place dans sa pensée. Jusqu'alors, Marie n'avait de l'admiration que pour les républicains:

Sa conscience lui criait bien que l'un se battait pour un homme, l'autre pour un pays; mais elle était arrivée par le sentiment au point où l'on arrive par la raison, à reconnâtre que le roi, c'est le pays ${ }^{26}$.

Ainsi, finalement, grâce à la «noblesse» de l'âme du nouveau chef, Montauran, encore jeune et donc idéaliste, l'image de l'Ancien Régime redevient positive. Le système rejeté par les Français présente une valeur certaine aux yeux de cette «républicaine», si précaire que soit le raisonnement qui justifie cette idée.

En effet, l'Histoire est bien le centre de ce roman «historique» et elle seule peut fournir une explication à la double personnalité, présentée avec insistance, comme très «bizarre» de Marie de Verneuil. Son instabilité, volontaire ou involontaire, vient du fait qu'elle est l'enfant de la République. Elle a été «mariée» à Danton, mais ce ré-

(22) Op. cit., p. 1027. Souligné par Balzac. 1ère éd.: «Etes-vous mademoiselle de Verneuil?... reprit-il d'une voix sourde et altérée» (p. 152).

(23) Op. cit., p. 1147. 1ère éd.: «Quand il m'aura sacrifié ses espérances, ses préjugés, le Roi et sa vie, peut-être aura-t-il réparé sa faute, et croirai-je alors être aimée!... Ah! Francine, dit-elle en se cachant le visage de ses mains, mon cœur s'emplit de compassion pour les souffrances de celle que je verrai douter de l'amour d'un homme! - Ah! flotter entre la haine et l'amour! Horreur!... - Il n'y a pas entre le paradis et l'enfer la place de poser mon pied» (p. 289).
(24) Op. cit., p. 1151 (p. 295)

(25) Op. cit., p. 1137 (p. 279).

(26) Op. cit., p. 1046. Il existe un petit changement par rapport à la version de 1829 , mais le sens est le même: «Sa conscience lui criait bien que l'un se battait pour un homme, l'autre pour un pays; mais l'amour lui persuadait que le bonheur du pays dépendait du système défendu par son amant» (p. 170). Cette phrase est capitale, et surtout le «mais» qui articule les deux parties: les deux termes de l'alternative ne s'opposent pas clairement, ne sont pas sur le même plan. 
publicain n'a eu qu'une vie extrêmement brève. Marie est devenue républicaine, mais pas encore de façon complète et totale: l'influence, toute extérieure, de Corentin, est plus forte qu'elle. Son choix pourrait se justifier par la conscience qu'elle a des défauts des royalistes, mais son avenir reste incertain. Son identité bâtarde est donc tout à fait représentative de la société française à la fin la Révolution française.

Mlle de Verneuil fait partie d'une nouvelle société qui veut supprimer son passé, son histoire et son patrimoine en espérant pouvoir ainsi se développer plus librement. Néanmoins, ce passage n'est pas facile et nécessite beaucoup de sacrifices, d'autant plus que son amour pour le chef royaliste signifie l'attachement de la nouvelle société au passé. La position de Marie est donc précaire, elle est celle d'une société moderne qui se cherche elle-même:

nos [c'est le narrateur qui parle de la société moderne] idées ne tiennent plus à rien, sont sans substance, sans point de départ, [...] le présent ne trouve plus de liens pour se rattacher au passé ni dans l'avenir. Tel fut l'état de Mlle de Verneuil. Penchée dans le fond de la voiture, elle y resta comme un arbuste déraciné ${ }^{27}$.

L'histoire d'amour entre Marie de Verneuil et le marquis de Montauran est donc complètement intégrée à l'Histoire. En racontant les épisodes d'une relation amoureuse impossible, c'est l'Histoire de la France que Balzac met en scène. L'instabilité de cette relation amoureuse est logique en cette époque de rupture et de changement. Marie, la France, ne peut pas devenir une autre du jour au lendemain, en éliminant tous ses aïeux; Montauran, de son côté, ne peut pas rester indifférent devant les sentiments ardents de la République, en tout cas pas toujours.

En fait, avant de devenir conscient de ses sentiments, se sentant humilié, le marquis a voulu combattre la République, en jetant cruellement Marie dans les bras du vilain Pille-miche. Mais, très vite, il regrette ce geste irréfléchi et il se trouve dans une position inférieure devant le succès remporté par la «citoyenne» Marie à son bal:

Elle reçut le jeune chef en boudant et sans mot dire, mais elle le quitta en souriant; elle le voyait si supérieur, qu'elle se sentit fière de pouvoir le tyranniser $[\ldots]^{28}$.

La République va devenir une réalité qui va fasciner Montauran avec laquelle il va avoir une relation passionnelle paradoxale, au lieu d'essayer de la détruire.

Ainsi, cette femme à la fois noble et républicaine est conçue comme le pivot de l'histoire; son identité bâtarde est le cœur de tous les problèmes de l'Histoire. En elle, toutes les questions posées aux deux partis politiques convergent; à travers elle, les antagonistes se rencontrent. L'Ancien Régime et la République arriveront-ils ainsi à trouver un moyen de s'accorder?

\section{Autour du pivot: le marquis de Montauran et le commandant Hulot}

La première rencontre entre les chefs des deux partis opposés se déroule dans une atmosphère assez tendue. Sur le plateau de la Pèlerine, apparemment, Montauran et Hulot dirigent d'une façon efficace leur armée. Les deux camps font preuve

(27) Op. cit., p. 1020 (p. 143).

(28) Op. cit., p. 1037 . $1^{\text {ere }}$ éd.: «Elle le reçut en boudant et sans mot dire, mais elle le quitta en souriant. Elle le sentait si supérieur, qu'elle ne résistait pas au plaisir de le tyranniser et de lui faire acheter bien cher de douces paroles même fausses» (pp. 279-280). Le marivaudage qui s'esquisse ici n'est qu'une apparence: Balzac ne perd pas de vue la dimension historique de l'œuvre. 
d'une force militaire quasi équivalente et tous deux sont confiants en leur chance de gagner. D'ailleurs, l'auteur dépeint dans le portrait de chaque chef la nature de chacun des deux partis. La qualité du stratège est en rapport avec celle de son parti politique: Montauran, c'est la «grâce» et Hulot, l'«énergie». Ils donnent ainsi une image en «contraste», tout en équilibre. Physiquement, ils n'ont rien en commun, mais leur représentation est aussi intéressante l'une que l'autre:

cet émigré [Montauran] une gracieuse image de la noblesse française; il contrastait vivement avec Hulot, qui, à quatre pas de lui, offrait à son tour une image vivante de cette énergique République pour laquelle ce vieux soldat combattait $[\ldots]^{29}$.

Néanmoins, juste après la «triste victoire» ${ }^{30}$, difficilement obtenue par les républicains, le narrateur commence à analyser les problèmes du parti royaliste. Tout d'abord, leur héroïque embuscade n'est en fait qu'un accident. Marche-à-terre a risqué sa vie en se mêlant à la troupe des Bleus, pour la surveiller afin que ses amis puissent «piller» tranquillement. Il n'avait donc pas pour but d'attaquer la troupe du commandant Hulot. Démasqué par ce dernier, il fait appel à des renforts pour protéger sa fuite. Ainsi, cette escarmouche n'a pas pour origine la défense «de Dieu et du Roi» ${ }^{31}$, ces «royalistes» n'ont que des objectifs de circonstance. Plus mercenaires qu'idéalistes, il leur faut, nécessairement, l'argent de ce «pillage» pour continuer leur combat contre la République. Leur «avidité» ne leur fait même pas déplorer la mort de cent camarades.

Parmi ces hommes, il n'y a pas que des paysans «sauvages», sans distinction sociale; on trouve également des gentilshommes qui sont devenus ironiquement des «gens-pille-hommes ${ }^{32}$ dans la bouche du patriote Cibot, dit Pille-Miche, envoyé par Marche-à-terre pour protéger la diligence. Voici le discours de Mme du Gua, une «affamée», discours entièrement approuvé par les chouans:

Vous avez perdu des hommes, nous n'en manquerons jamais. Le courrier porte de l'argent, et nous en manquerons toujours! Nous enterrons nos hommes qui iront au ciel, et nous prendrons l'argent qui ira dans les poches de tous ces braves gens. Où est la difficulté? ${ }^{33}$

Non seulement Montauran est complètement étranger à la décision de procéder à «sa» première attaque, mais en plus, après avoir enfin compris l'intention de ses hommes, il a beaucoup de mal à s'imposer. Il manifeste son mécontentement et tente d'éveiller leur fierté de combattants:

Comment! s'écria le jeune homme en colère, c'est donc pour arrêter une voiture que vous restez encore ici, lâches qui n'avez pu remporter une victoire dans le premier combat où j'ai commandé! $!^{34}$

(29) Op. cit., p. 936. L'opposition entre les adjectifs «gracieuse» et «énergique» est d'une remarquable pertinence. L'idée d'une force égale entre les deux troupes était encore plus claire en 1829: «La victoire aurait pu rester indécise pendant des heures entières, ou la lutte se serait terminée faute de combattans, car bleus et chouans montraient un courage égal», (p. 51) L'édition établie par Andrew Olivier tient à respecter les conventions typographiques et orthographiques de l'époque. Il faut également noter l'inversion significative des rapports entre un royaliste qui a toute la beauté de la jeunesse, et un «vieux soldat» qui représente l'avenir.

(30) Op. cit., p. 941.

(31) Ibid. (p. 57).

(32) Op. cit., p. 948. Bien que ce jeu de mot ironique n'existe pas encore dans l'édition de 1829 , nous y trouvons déjà l'opposition entre les gentilshommes d'un côté, les «pilleurs» et les «voleurs» de l'autre.

(33) Op. cit., p. 944 (p. 58).

(34) Op. cit., p. 941 (p. 57). 
Cependant, sa «colère» ne va pas le pousser plus loin. Au lieu de s'opposer directement à ses chouans, il préfère éviter le problème; il s'absente exprès pour ne pas voir ses hommes plus intéressés par l'or que par le rétablissement de la monarchie: «En me retirant, je puis ignorer cette lâcheté» ${ }^{35}$. Faute de pouvoir donner un ordre respectable et respecté, Montauran essaie de «sauver l'honneur de [son] pouvoir» ${ }^{36}$. Il aurait peut-être voulu «sanctionner» les «pillards» pour de «tels brigandages», mais, d'abord, il ne s'en sent pas capable ${ }^{37}$, et puis sa propre vie lui semble plus importante: «Écoutez-moi [dit-il à Mme du Gua], reprit-il après une pause, si je demeurais là pendant la capture de cette diligence, nos gens me tueraient...» ${ }^{38}$.

Pour essayer de faire comprendre la gravité de cet acte infâme à ses royalistespillards, Montauran n'a pas trouvé d'autre solution que de leur parler sur le même ton, en se servant de l'appât de la «récompense». La noblesse spirituelle est donc mise de côté:

Ceux qui désormais se rendront coupables d'attaques si honteuses ne recevront pas l'absolution et ne profiteront pas des faveurs réservées aux braves serviteurs du Roi ${ }^{39}$.

À la tête d'un groupe d'hommes qu'il ne comprend pas, en tout cas qui ne le comprennent pas, qu'il n'apprécie guère, le jeune chef royaliste a bien des difficultés à commander: «Il était facile de voir que l'autorité du nouveau chef, si difficile à établir sur ces hordes indisciplinées, allait être compromise» ${ }^{40}$.

En effet, la situation de Montauran est très difficile. Non seulement l'argent est la principale préoccupation de la majorité de ses hommes, mais en plus c'est un groupe hétérogène. Les «gentilshommes» ne se battent que pour la «récompense» ${ }^{41}$, les chouans ne sont que des «bêtes ${ }^{42}$ ou des «barbares» ${ }^{43}$ à côté des Vendéens qui se montrent plus «nobles» ${ }^{44}$ et plus «réguliers». Comment Montauran peut-il se reconnaître parmi ces gens? Surtout quand il réalise qu'il n'a plus grand chose en commun avec la plupart des «gentilshommes» de son rang et que les chouans «indisciplinés» ne pourront jamais «comprendre et exécuter [ses] ordres» ${ }^{45}$.

La scène «assez étrange» ${ }^{46}$ où les nobles exigent leur «salaire» ${ }^{47}$ de Montauran montre bien cette rupture au sein du parti royaliste. S'ils n'obtiennent pas «l'argent promis au nom du Roi» ${ }^{48}$, les gentilshommes ne reconnaîtront plus «l'autorité de personne». Sans récompense, ils «quitter[ont] le service du roi» ${ }^{49}$ pour aller «traiter avec le premier Consul» $\aleph^{50}$. Même les prêtres ne s'occupent plus de leur Dieu. Pour avoir quelque reconnaissance de ses manipulations auprès des villageois superstitieux, l'ab-

(35) Ibid. Cette phrase n'est pas dans la version de 1829 mais la déception de Montauran est immense lorsqu'il s'interroge sur sa position de «chef»: «Suis-je chef...?» (p. 56).

(36) Op. cit., p. 945.

(37) «Je ne saurais sanctionner de tels brigandages par ma présence», op. cit., p. 944 (p. 59).

(38) Op. cit., p. 945. Les points de suspension sont de l'auteur. Mais on peut supposer que ce sont des propos de Montauran contre le pillage. Dans la version de 1829 , Montauran ne prononce qu'un mot: «adieu», avant de disparaître (p. 59).

(39) Op. cit., p. 943 (p. 57).

(40) Ibid. Dans l'édition de 1829, Balzac parle de la «douteuse autorité» (p. 58) de Montauran. Son hostilité est interprétée par Mme du Gua comme une intention de «s'arranger à l'amiable avec la République!» (p. 59).

(41) Op. cit., p. 1127 (p. 267).
(42) Op. cit., p. 1060 (p. 187).

(43) Ibid. «Ces soldats de nouvelle espèce et sur lesquels se fondaient les espérances de la monarchie [...] sept ou huit d'entre eux s'amusaient à lancer dans les eaux les cadavres des Bleus auxquels ils attachaient des pierres. Ce spectacle, joint aux différents tableaux que présentaient les bizarres costumes et les sauvages expressions de ces gars insouciants et barbares, était si extraordinaire et si nouveau pour $M$. de Fontaine [...]» Dans la version de 1829, le chef des Vendéens était M. de Châtillon.

(44) Ibid.

(45) Ibid. (p. 187).

(46) Op. cit., p. 1125 (p. 266).

(47) Op. cit., p. 1127 (p. 267).

(48) Op. cit., p. 1126 (p. 266).

(49) Ibid. (p. 266).

(50) Op. cit., p. $1129.1^{\text {ère }}$ éd.: «je me rends...» (p. 269). 
bé Gudin s'est volontairement fait «l'ambassadeur» de ces «calculateurs» ${ }^{51}$ pendant leur révolte. Ils n'ont qu'une pensée en commun: soumettre un chef inexpérimenté. C'est donc un complot «convenu» entre tous les membres royalistes contre Montauran, qui se retrouve exclu de son propre parti.

Ces discussions montrent la gravité du problème: les nobles ne se sentent pas concernés par leur guerre contre la République. Dans leurs combats, ils ne voient qu'un Roi détrôné; ceux qui ont été guillotinés sont déjà oubliés. Par conséquent, la politique de réconciliation de Bonaparte avec une partie des nobles de l'Ancien Régime est une bonne occasion pour ceux qui ont des vues courtes et qui veulent en tirer des profits personnels. Car les seigneurs d'Ancien Régime, s'ils le demandent, pourront rester seigneurs sous Bonaparte; ils garderont tous les privilèges que le Roi leur reconnaissait. Ainsi, la «peine» qu'ils se donnent pour rétablir le Roi «mérite» bien une «pension» ou une compensation quelle qu'elle soit. Pour se faire pardonner leur honteuse avidité, les nobles trouvent une excuse en parlant de leur dette et de leur «créancier diablement importun [...] qu'il faut toujours satisfaire!» ${ }^{52}$. L'argent, qu'il vienne du Roi ou du premier Consul, ne fait aucune différence pour eux.

Face à ces demandes d'un «prix exagéré»" ${ }^{53}$, Montauran est forcé de répondre immédiatement et ne peut pas traiter le problème «trop légèrement $»^{54}$. Sa position est donc «très délicate» ${ }^{55}$ entre les attitudes tantôt humbles et tantôt menaçantes de ses troupes qui réclament sans scrupule «dès le matin le prix de la journée» ${ }^{56}$. De plus, parmi ces hommes qui lui répugnent, il lui est bien difficile de reconnaitre ceux qui ont la même vocation que lui, qui voit «un Roi dans le Roi, et non une proie à dévorer» ${ }^{57}$. Après un examen attentif, le jeune chef ne trouve que deux militaires loyaux dans cette bande, un noble et un chouan: le baron du Guénic et le major Brigaut. Sa désillusion va ainsi jusqu'à lui donner l'idée de se séparer de ces profiteurs, qui ne voient en lui qu'un intermédiaire entre eux et la récompense promise par le Roi. En détruisant la lettre patente du roi, Montauran s'apprête à renoncer à son autorité et à rendre la «liberté» à ses militaires vénaux, avec lesquels il ne veut plus se battre: «Vous êtes libres, messieurs, de m'abandonner...»»

Même si, grâce aux scrupules de quelques proches, le marquis parvient à rester à la tête de sa troupe, il se sentira toujours différent de ces «royalistes»:

Le marquis essaya vainement de quitter l'air sombre qui altéra son visage, les chefs aperçurent aisément les impressions défavorables que cette scène avait produites sur un homme dont le dévouement était encore accompagné des belles illusions de la jeunesse, et ils en furent honteux ${ }^{59}$.

Pour ces royalistes, Montauran est décidément un chef encore trop jeune ${ }^{60}$, un étranger. Voici les critiques du comte de Bauvan devant Marie de Verneuil:

(51) Op. cit., p. 1127 (p. 267).

(52) Ibid. (p. 267).

(53) Op. cit., p. 1128. $1^{\text {ère }}$ éd.: «des prétentions aussi exagérées» (p. 270).

(54) Ibid. (p. 267).

(55) Op. cit., p. 1130 (p. 270).

(56) Op. cit., p. 1131. $1^{\text {ere }}$ éd.: «dès le matin le prix d'une journée» (p. 271).

(57) Op. cit., p. 1130 (p. 271).

(58) Ibid. (p. 271).

(59) Op. cit., p. 1131. $1^{\text {ère }}$ éd.: «Le marquis es- saya de déposer l'air sombre et la mélancolie qui altéraient son visage; mais les chefs, honteux de la scène qui venait d'avoir lieu, aperçurent aisément les défauts du masque qu'il s'efforçait de garder» (p. 271).

(60) Voir ci-dessus la note 29 et la p. 936 des Chouans (le mot grâce n'a évidemment pas le même sens). Dès le début du roman, le jeune âge de Montauran est déjà souligné par le narrateur. Hulot ne donne «pas plus de vingt-cinq ans» à son adversaire. Op. cit., p. 935 (p. 48). 
Il [Montauran] est trop jeune pour avoir vu Versailles. Son éducation a été manquée, et au lieu de faire des noirceurs, il donnera des coups de couteau. [...] Oui, quoique ce soit un homme à bonnes fortunes, il n'en a ni le laisser-aller, ni la grâce ${ }^{61}$.

Ainsi, il ne fait aucun doute que les quelques repentis difficilement ralliés par la sincérité de Montauran ne tiendront pas plus longtemps que leur fragile loyauté envers le Roi.

Enfin si, malgré tout, Montauran se bat toujours du côté royaliste, il est sûr qu'il n'a plus la même «conviction»; il ne continue son combat que pour lui-même:

«Mais ce jeune homme avait trop d'élévation dans l'âme, trop d'orgueil, trop de conviction peut-être pour délaisser l'œuvre commencée, et il se décidait en ce moment à la poursuivre courageusement malgré les obstacles» ${ }^{62}$.

S'il avait eu le courage de renoncer à son amour pour Marie par fidélité à son parti, à présent, sa déception politique et religieuse va le pousser à l'extrême de ses propres sentiments. Il n'hésite plus à faire des reproches, face à face, à l'abbé Gudin qui invoque la «Foi» et se sert de la Religion pour satisfaire ses ambitions personnelles:

Me prenez-vous pour un idiot avec votre sermon sur la danse, reprit Montauran. Ne figureriez-vous pas de bon cœur dans une chaconne pour vous [l'abbé Gudin] retrouver rétablis sous votre nouveau nom de Pères de la Foi!... ${ }^{3}$.

Le marquis est donc forcé d'abandonner sa fonction de chef; il se sépare des nobles et des prêtres pour qui l'honneur et la foi ne sont que des prétextes. Cette déception va jusqu'à le pousser à commettre la «sottise» ${ }^{64}$ d'épouser la républicaine:

le marquis absorbé dans sa rêverie croyait entendre quelques-unes des raisons que, d'une voix prophétique, Marie lui avait données au milieu de ces mêmes chefs à la Vivetière, pour l'engager à abandonner la lutte des rois contre les peuples ${ }^{65}$.

Ainsi, la rupture entre Montauran et son parti ne peut pas être considérée comme une faute. Car c'est le parti royaliste qui est coupable. De graves problèmes intérieurs à son camp ébranlent la conviction de son jeune chef, qui reconnaît ainsi l'impossibilité et surtout l'inutilité de sa tâche. Le rétablissement du Roi n'est plus défendable lorsque les nobles ne sont plus dans leur majorité que des «calculateurs», et l'armée royale qu'une bande de mercenaires indisciplinés.

L'adversaire du marquis, le commandant Hulot, le fier représentant de la République, ne ressasse visiblement pas non plus que des idées hostiles contre l'Ancien Régime. Face au «large cordon rouge» ${ }^{66}$, symbole de la royauté, porté par le marquis, Hulot se laisse distraire momentanément par cette gloire du passé, avant de revenir à la réalité, avec «sa petite troupe»:

(61) Op. cit., p. 1107. La dernière phrase de cette citation n'a été rajoutée qu'après 1829 (p. 242).

(62) Op. cit., p. 1132. $1^{\text {ere }}$ éd.: «Mais ce jeune homme avait trop d'élévation dans l'âme, trop d'orgueil et peut-être trop de conviction pour délaisser l'œuvre de sa pensée. Il se décidait en ce moment à poursuivre courageusement son entreprise» (p. 272).

(63) Op. cit., p. 1089. 1ère éd.: «Me prenez-vous, continua-t-il, pour un idiot avec votre danse, et ne figureriez-vous pas de bon cœur dans une chaconne pour vous retrouver à Juvigny» (p. 221).
(64) Op. cit., p. 1205. Comme pour Felipe de Macumer, la politique et l'amour sont les deux choses les plus importantes pour l'«émigré». La version de 1829 est explicite: «Ah! le cri du pauvre émigré est complet: Dieu, le Roi et ma dame!» (p. 139).

(65) Op. cit., p. 1132. $1^{\text {ere }}$ éd.: «le marquis croyait encore entendre la voix prophétique de mademoiselle de Verneuil, lorsque, dans le salon de la Vivetière, elle l'engageait à abandonner la lutte des rois contre les peuples» (p. 272).

(66) Op. cit., p. 935 (p. 48). 
Les yeux du commandant, attirés d'abord par cette royale décoration, alors complètement oubliée, se portèrent soudain sur un visage [celui de Montauran] qu'il perdit bientôt de vue, forcé par les accidents du combat de veiller à la sûreté et aux évolutions de sa petite troupe $^{67}$.

Revenant sur son adversaire, Hulot est «frappé» et «attiré» par les qualités spécifiquement nobles de Montauran. Pour Hulot, le marquis est incontestablement un «militaire», bien que sa façon de se battre soit particulière: son attitude hors du commun est «à la manière de ceux qui veulent dans un combat une certaine poésie de convention» ${ }^{68}$; sa «contenance accusait tout à la fois de l'élégance et de la force» ${ }^{69}$. Avec ses «mains bien gantées», sa peau «blanche», ses traits «délicats» et sa blondeur éblouie sous le soleil, l'expression gracieuse du marquis est en parfaite harmonie avec sa personne. Enfin, la «poésie» de ce jeune «militaire cravaté» révèle une prestance que le «brave» général napoléonien n’atteindra jamais.

La «grâce» de la noblesse française inspire donc paradoxalement du respect à l'officier républicain, même si son caractère trempé résiste. À l'auberge des TroisMaures, à Alençon, devant le prétendu élève de l'Ecole Polytechnique qui a du mal à aller jusqu'au bout de ses mensonges en disant qu'il est du corps de la «marine», un corps qui n'existe pas, et qui prétend se nommer Gua Saint-Cyr, Hulot respecte l'anonymat maladroit de son adversaire. Bien que Hulot soit sûr de la fausseté de cette identité, il tient à affronter son ennemi sur le champ de bataille avec honneur. Ainsi, parallèlement à l'intervention de Marie, le commandant laisse tranquillement s'enfuir l'homme et sa prétendue «mère» visiblement trop jeune. Hulot va même jusqu'à «s'excuser» de ses «soupçons» qui sont pourtant bien fondés, en acceptant le verre offert par Montauran: «Le brave officier offrait sur sa figure embarrassée l'expression de la lutte que la sévérité de ses devoirs livrait dans son cœur à sa bonté naturelle» ${ }^{70}$. Passage important qui souligne une «bonté» qui contredit son «devoir». Il doit arrêter le marquis mais, comme ce dernier, il obéit au même code d'honneur. $\mathrm{Au}$ fond, Hulot est très sensible au patrimoine historique malgré sa position politique, tout comme Balzac lui-même ${ }^{71}$.

L'attitude plutôt respectueuse de Hulot paraît encore plus clairement si nous la comparons avec celle du policier Corentin:

Si c'est là un républicain, se dit Corentin en le voyant sortir, je veux être pendu! [...] Et si c'est là sa mère, se dit-il encore en regardant Mme du Gua, je suis le pape! ${ }^{72}$

Marie revient du bal et elle a résolu de «se venger» du marquis; Hulot et Corentin n'ont pas les mêmes réactions. Le commandant se présente à Marie, certes, pour des raisons professionnelles, mais il est aussi venu pour s'assurer de son état; ses inquiétudes se traduisent sur sa figure «soucieuse» et «interrogative» ${ }^{73}$. En revanche, Corentin se comporte en enquêteur. Pour pouvoir surprendre la vraie pensée de son

(67) Op. cit., pp. $935-936$ (p. 48).

(68) Op. cit., p. 936 (p. 48). «Poésie de convention»: paraphrase exacte de cette «grâce» que le narrateur prête à Montauran.

(69) Ibid. (p. 48).

(70) Op. cit., p. 991 (p. 111).

(71) Nous savons qu'au moment où l'auteur rédige cette œuvre (1828), il est plutôt du côté républicain, mais bientôt, il deviendra plus royaliste que le roi. La troisième édition des Chouans a subi de ce fait en 1845 des modifications très significatives, notamment le passage des réflexions de Marie sur l'opposition entre l'idéal de Montauran et celui de l'officier républicain Gérard. (voir éd. Pl. pp. 869870) D'après L. FrappIER-Mazur («Introduction» aux Chouans, CH., Pléiade, t. VIII), les opinions beaucoup plus «tranchées» de Marie sont celles d'un Balzac «royaliste».

(72) Op. cit., p. 980 (p. 99).

(73) Op. cit., p. 1147. 1ère éd.: «très-interrogative» (p. 290). 
interlocutrice, il essaie d'obtenir des informations que cacherait Marie en feignant une certaine «négligence», pour dissimuler ses regards «obliques». En effet, Corentin ne s'intéresse pas aux réponses de Marie, mais à sa façon de répondre.

Même face à la femme qu'il cherche à séduire, Corentin ne peut pas s'empêcher d'être méprisant et provocateur. En plus, il s'est même déjà informé avant sa visite:

Il paraît que votre pouvoir a cessé là [aux portes de Fougères], reprit Corentin, et que la peur du ci-devant [Montauran] surpasse encore l'amour que vous lui inspirez ${ }^{74}$.

Le comportement désagréable de Corentin continue de s'opposer à la noblesse de l'officier. Lorsque Hulot s'aperçoit de l'hésitation de Marie sur sa décision de vengeance, non sans étonnement, il réagit d'une façon calme et extrêmement galante:

Le vieux soldat s'avança vivement vers elle et lui prit la main pour la baiser, avec une sorte d'enthousiasme; puis, il la regarda fixement et lui dit d'un air sombre: - Vous oubliez mes deux amis et mes soixante-trois hommes ${ }^{75}$.

D'ailleurs, face à cet amour de la républicaine pour le chef royaliste, le général fait toujours preuve de discrétion et de respect. Sans comprendre précisément les sentiments et les mensonges de Marie, il observe une rigoureuse neutralité.

Cette différence fait que les deux hommes qui sont au service de la République n'ont pas les mêmes manières d'agir. Les pensées et les actions de Hulot sont toujours en accord; il exécute scrupuleusement les ordres ordonnés. En revanche, l'«espion» Corentin, sous la tutelle du ministre de la police, dissimule ses idées, ses calculs, et se sert «habilement [des] passions des hommes ou des femmes comme des ressorts que l'on fait mouvoir au profit de l'Etat $[\ldots] \gg^{76}$. Son plus grand plaisir est de détecter et d'utiliser «les plus indomptables sentiments comme des détentes» ${ }^{77}$ pour se mettre ainsi à la place de «Dieu». Hulot affronte les ennemis «en face», Corentin, lui, préfère les poignarder $\left\langle\right.$ par derrière» ${ }^{78}$. Enfin, toutes les petitesses de l'espionnage finissent par provoquer un «profond dégoût» chez le commandant, qui tâche dès lors de se distinguer de ce «renard»:

Si le premier Consul m'écoutait [...] il laisserait ces renards-là combattre les aristocrates, ils sont dignes les uns des autres, et il emploierait les soldats à toute autre chose ${ }^{79}$.

Une guerre, d'après l'officier, ne se gère pas avec des calculs sournois; les moyens honteux déshonorent les soldats, qui préfèrent perdre leur vie glorieusement au cours d'une bataille. Une victoire sans gloire n'est qu'une frustration pire que la mort. Ainsi, le militaire s'oppose au policier. La République pour laquelle ils se battent ensemble n'est visiblement pas la même pour tous. Hulot croit sincèrement aux principes de la société républicaine; Corentin n'y voit qu'un moyen de parvenir à ses fins ${ }^{80}$. Dé-

(74) Ibid. 1ère éd.: «Mais là, reprit Corentin, votre pouvoir a cessé... L'amour de notre ci-devant n'est pas encore plus fort que sa peur» (p. 290).

(75) Op. cit., p. 1147. Ce comportement extrêmement galant peut paraître surprenant chez un «brave» commandant qui est présenté comme totalement étranger aux «manières» artificielles de la cour. Mais ce noble comportement confirme le contraste entre Hulot et Corentin. D'ailleurs, cette réaction extrêmement courtoise de Hulot n'a été rajoutée que plus tard; dans la version de 1829, il ne répondait qu'avec une «petite grimace» (p. 290).

(76) Op. cit., p. 1148 (p. 292).

(77) Ibid. (p. 292).

(78) Ibid. (p. 292).

(79) Op. cit., p. 1149. (p. 293) C'est une opinion historiquement attestée (certains généraux répugnaient d'être envoyés combattre d'autres Français).

(80) Corentin, muscadin-policier formé par Peyrade, devient le chef de son maître dans Splendeurs et Misères des courtisanes: «Corentin, beaucoup 
calage qui est d'ailleurs comparable avec la situation de Montauran au milieu de ses royalistes. Hulot prend ainsi la décision de défendre le droit de tous les guerriers; peu importe à qui va leur fidélité, l'«honneur» de mourir sur le champ de bataille doit être protégé:

Hulot résolut-il de contrarier Corentin en tout ce qui ne nuirait pas essentiellement aux succès aux vœux du gouvernement, et de laisser à l'ennemie de la République les moyens de périr avec honneur les armes à la main, avant d'être la proie du bourreau de qui ce sbire de la haute police s'avouait être le pourvoyeur ${ }^{81}$.

La distinction entre les soldats royalistes et républicains est superficielle et n'existe donc plus pour Hulot. Ce qui compte, c'est la gratuité et l'engagement de chacun. L'esprit «guerrier», c'est-à-dire «noble», passe avant tout.

Par conséquent, d'une certaine façon, le marquis de Montauran et le général Hulot arrivent à trouver un terrain d'entente et les deux systèmes politiques ne sont plus si opposés. Tous les deux, idéalistes et sincères, en butte aux problèmes intérieurs de leur propre parti, commencent à se rapprocher. D'ailleurs, la grandeur des nobles idées de Hulot ne sont-t-elles pas une preuve de sa «noblesse»? Tous les deux arrivent ainsi, chacun à sa manière, à incarner le même esprit noble. En se rapprochant chacun de son adversaire, Montauran et Hulot reconnaissent chez l'autre une valeur commune.

Ainsi, sûr de la mort du marquis, Hulot se montre «attentif et chagrin» ${ }^{82}$. Avant de mourir, c'est à Hulot que le marquis demande un dernier service. Un testament qui témoigne d'ailleurs de la sympathie de Montauran pour les Français, pour la République et bien sûr, pour l'officier:

Commandant, dit le marquis en rassemblant toutes ses forces et sans quitter la main de Marie, je compte sur votre probité pour annoncer ma mort à mon jeune frère qui se trouve à Londres, écrivez-lui que s'il veut obéir à mes dernières paroles, il ne portera pas les armes contre la France, sans néanmoins jamais abandonner le service du Roi ${ }^{83}$.

Pour répondre à cette ultime marque d'estime du marquis, Hulot chasse Corentin, «le roi des espions» ${ }^{84}$, dont la présence leur est devenue insupportable. Ainsi, Montauran n'est plus l'adversaire de Hulot; la République ne s'oppose plus à l'Ancien Régime. Les deux soldats, ennemis sur le champ de bataille, ont des ennemis «redoutables» en commun: les policiers-espions, qui sont au fond de vrais criminels ${ }^{85}$ :

plus fort que Peyrade d'ailleurs, quoique plus jeune, et qui ne fut un homme de génie que dans les souterrains de la police» (CH., t. VI, p. 530). Ses manœuvres restent toujours obscurs, car il n'a qu'un principe: tirer un profit personnel de son activité professionnelle: «Corentin, resté l'un des généraux dont le Ministre de la police est le Connétable avait gardé, sous le duc de Rovigo, la place éminente qu'il occupait sous le duc d'Otrante. Or, il en était alors de la Police Générale comme de la Police Judiciaire» (Op. cit., p. 533).

(81) Les Chouans, cit., p. 1149. 1ère éd.: «Il s’intéressa peut-être plus à son ennemi qu'à mademoiselle de Verneuil, et résolut de contrarier Corentin en tout ce qui ne nuirait pas essentiellement aux succès et aux vœux du gouvernement. Il se promettait intérieurement de chercher toutes les occasions de laisser à l'ennemi de la république le moyen de périr honorablement, avant d'être la proie du bourreau dont Corentin se faisait le pourvoyeur» (p. 293).

(82) Op. cit., p. 1208 (p. 363 ).

(83) Op. cit., p. 1210 . Cet acte significatif de la transmission du pouvoir n'a été ajouté qu'après 1829.

(84) Op. cit., p. 1157 (p. 301).

(85) Cf. Splendeurs et Misères des courtisanes, où la position de Corentin est problématique. Par opposition à Vautrin, il n'est pas un «vrai» criminel. Évidemment, cet être «dégoûtant», «répugnant», sans aucune valeur humaine, n'est pas non plus du côté des «grands» et des nouveaux dirigeants de la société; il n'agit que par intérêt. Ses relations avec Marie, la bâtarde, restent toujours floues. Qu'est-ce 
Hulot prit l'espion par le bras, de manière à lui laisser l'empreinte de ses ongles dans la chair, et lui dit: - Puisque ta besogne est finie par ici, fiche-moi le camp [...]

«Et déjà le vieux soldat tirait son sabre» ${ }^{86}$.

Après «un signe de tête» du marquis pour remercier le commandant de cet acte hautement emblématique, ces deux hommes ne sont plus de simples ennemis, mais de «loyaux ennemis» ${ }^{87}$.

Le respect, l'estime et même l'admiration entre deux partis politiques opposés sont donc une chose possible pour Balzac. C'est Hulot qui est désigné pour recueillir la dernière parole du marquis mourant et pour la transmettre à son frère. C'est donc le soldat «républicain» qui s'occupera de la vie d'un «noble» ${ }^{88}$, d'un autre Montauran. Après avoir reconnu et surtout retenu les qualités «nobles» de l'Ancien Régime, c'est la République qui assurera leur survie. Ainsi, l'idée de rupture n'existe pas dans Le Dernier Chouan ou la Bretagne en 1800. Le changement politique est davantage fondé sur le «mariage» que sur les armes. Le général n'a pas tué le marquis de ses propres mains; d'ailleurs, d'une certaine façon, c'est Montauran lui-même qui a décidé d'offrir sa vie à la «République»: «Marie, ma vie est à vous».

\section{«Un jour sans lendemain»: une vision tragique et optimiste}

Montauran et Marie ont décidé ensemble de leur destin. L'amour du marquis est une vraie renaissance, celle dont rêvait Marie avant de rencontrer Montauran. Pour elle, la mort n'a rien d'effrayant parce qu'elle a «cueilli les fleurs» ${ }^{89}$ de la vie, parce qu'elle a «vécu». Ainsi, lorsqu'elle est sûre d'être «aimée» et après avoir «reconquis l'estime de l'homme qui représente à [ses] yeux le monde entier» ${ }^{90}$, la mort n'est que «bonheur» pour elle. De ce fait, bien que ces deux amants perdent leur vie le lendemain de leur mariage, on doit constater la vision très positive de l'auteur. Cette union finale qui réussit à se concrétiser et même à se consommer malgré tous les obstacles, n'est-elle pas une preuve de l'optimisme balzacien? La rencontre entre l'Ancien Régime et la République n'est pas vouée à l'échec, elle recèle de l'«espoir».

D'après l'auteur, même si les préparatifs religieux du mariage sont «bizarres» ${ }^{91}$ à cause de leur «triste mémoire», ils restent «touchants». D'ailleurs, à cause de la

donc que cette police? Quelle est sa force sociale, si elle n'est ni criminelle, ni noble, ni bourgeoise? Sa situation devient encore plus opaque dans Splendeurs et Misères des courtisanes, où il continue son aventure et où Vautrin accepte de devenir le chef de la police secrète. La vision de Balzac sur la police nous semble donc peu claire dans la construction d'une nouvelle société. Pourtant, pour M. ANDRÉOLI (Lecture et mythes: "Les Chouans" et "Les Paysans" d'Honoré de Balzac, Paris, Honoré Champion Éditeur, Paris, 1999, p. 189) Corentin est bien un homme de son temps: «il est le seul à ne jamais parler de passé ni d'avenir: car ce jeune homme, inclassable jusque dans ses vêtements hybrides, personnifie la structure du système, intemporel en son essence, et pour ainsi dire impartial». On pourrait dire, plus justement peut-être, cynique.

(86) Ibid. (p. 366) Dans la version de 1829, le roman se termine sur la réaction de Corentin: «Voilà encore un de ces honnêtes gens qui ne feront jamais fortune, répondit Corentin - quand il fut hors du corps-de-garde». Bien que Balzac n'insiste pas encore sur l'échange entre Hulot et Montauran juste avant la mort de ce dernier, il est évident que le caractère et les comportements nobles de Hulot le désignent comme le successeur politique du marquis, de l'Ancien Régime. Ainsi, l'insertion de ce passage décisif témoigne de la volonté de «se commenter» de Balzac lors de ses reprises du roman; la dernière version des Chouans précise sans doute mieux ses idées de 1829.

(87) Op. cit., p. 1211.

(88) Cette situation sera expliquée davantage à la fin de La Cousine Bette, lors de la mort du général Hulot.

(89) Op. cit., p. 969 (p. 86).

(90) Op. cit., p. 1140. Ces propos ne figurent pas dans l'édition de 1829.

(91) Op. cit., p. 1205. (p. 359) Nous remarquons le même adjectif lorsque l'auteur décrit Mlle de Verneuil. Tout ce qui relève du mélange, qui est difficile à définir, est qualifié de «bizarre». 
«grâce» et des «mystères» de ces cérémonies, le pouvoir de la Religion ne pourra jamais être détruit. Ainsi, ce mariage béni par «Dieu» est protégé par la force divine et compense les «discordes civiles». Dans peu de temps, grâce à la puissance incommensurable de la Religion dont la principale fonction est de réunir les différentes forces sociales, les Français se réconcilieront une fois de plus. La légitimité de ce mariage est donc parfaite, totale et incontestable:

L'union du marquis et de Mlle de Verneuil allait être consacrée, comme tant d'autres unions, par un acte contraire à la législation nouvelle; mais plus tard, ces mariages, bénis pour la plupart au pied des chênes, furent tous scrupuleusement reconnus ${ }^{92}$.

La Religion joue donc un rôle de réconciliation très significatif dans ce roman ${ }^{93}$. Pour pouvoir sauver la vie de Montauran, à la vue du prêtre, la républicaine change brutalement d'attitude et se met à croire en Dieu «avec un incroyable enthousiasme»:

Mlle de Verneuil se précipita à genoux avec un incroyable enthousiasme: $\mathrm{O}$ mon Dieu! dit-elle dans son extase, ma foi en toi est égale à mon amour pour lui! inspire-moi! Fais ici un miracle ou prends ma vie ${ }^{94}$.

Or, jusque là, elle se montrait plutôt distante et critique face à la manipulation de l'abbé Gudin" ${ }^{95}$ qui pourrait être une figure symétrique de celle de Corentin. D'ailleurs, l'image de ce «gros» ${ }^{96}$ abbé, homme $\left\langle\right.$ redoutable» ${ }^{97}$ qui parle d'une voix «forte» et dont «la violence alla toujours en croissant» ${ }^{98}$, s'efface devant cette nouvelle figure de la religion.

En effet, la religion elle-même a subi un profond changement dans ce contexte politique. Le réalisateur de cette «union» impossible est devenu lui-même le symbole de cette réconciliation longtemps espérée. À travers lui et grâce à la réussite de cette synthèse, Balzac entrevoit un retour vers la «paix»:

Le prêtre qui conservait ainsi les anciens usages jusqu'au dernier moment, était un de ces hommes fidèles à leurs principes au fort des orages. Sa voix, pure du serment exigé par la République, ne répandait à travers la tempête que des paroles de paix ${ }^{99}$.

Devant cette religion tolérante, en répondant «oui» à son bonheur et à son malheur, résolue désespérément à mourir à la place de son mari, la nouvelle croyante, Marie, est devenue une «sainte» ${ }^{100}$. Même si sa vie ne s'échangera pas contre celle de

(92) Op. cit., p. 1205. 1ère éd.: «la législation adoptée» (p. 359).

(93) Ce n'est pas n'importe quelle religion, pas celle de Gudin, mais une religion mystique qui est celle des «druides». Le prêtre qui manipule ses croyants n'est pas un prêtre authentique. Mais la notion de religion naturelle reste floue dans le roman.

(94) Op. cit., p. 1204 (p. 359). Toute cette scène doit être effectivement interprétée comme un «miracle» et ce mot peut être défini précisément comme improbabilité maximale d'une part, et d'autre part comme sublime synthèse des différents niveaux du réel: le niveau individuel (sexe, sentiment procréation: mariage), le niveau politique (au paroxysme de son déchaînement: la guerre), le niveau religieux (le prêtre-druide représentant la transcendance divine). L'épisode est à la fois tragique et glorieux.
(95) «- Francine! lui dit-elle [Mlle de Verneuil] à voix basse, tu as donc peur d'être une Mahumétische? [...] L'attitude de Francine annonçait une conviction si profonde, que Marie comprit alors tout le secret de ce prône, l'influence du clergé sur les campagnes, et les prodigieux effets de la scène qui commença», op. cit., p. 1120 (p. 260).

(96) Op. cit., p. 950 (p. 65).

(97) Op. cit., p. 1036. «Habitué [l'abbé Gudin] à se servir de la religion comme d'un instrument, il persuade à ses affiliés qu'ils ressusciteront, et sait entretenir leur fanatisme par d'adroites prédications [...] il faut employer les intérêts particuliers de chacun pour arriver à un grand but. Là sont tous les secrets de la politique» (p. 159).

(98) Op. cit., p. 1118 (p. 257)

(99) Op. cit., p. 1205 (pp. 359-360).

(100) Op. cit., p. 1206. «La voilà une sainte, dit 
Montauran, qui va mourir en même temps qu'elle, leur mort apporte le calme et la «paix» à tous: Francine n'aura plus de remords à aimer Marche-à-terre ${ }^{101}$; le fils de Galope-Chopine pourra grandir paisiblement, sans vouloir venger son père; et enfin, le caporal Gudin ne sera pas «déshérité» ${ }^{102}$ par son oncle, le riche abbé Gudin.

Par conséquent, la mort de ces deux personnages est bien une «tragédie» ${ }^{103}$ mais une tragédie qui n'exclut pas l'espoir. Au contraire, elle présage une fusion entre les deux partis politiques antagonistes. Car, bien que l'amour passionné entre Montauran et Marie arrive à survivre dans de dures conditions - «L'amour est la seule passion qui ne souffre ni passé ni avenir» ${ }^{104}-$ leur mariage est définitivement ancré dans le «présent». Ainsi, pour que soit construit un «avenir» pacifié, ce passage par la mort est jugé inévitable. Car il n'y a que la Mort qui permette au système social de se renouveler; il faut détruire pour pouvoir construire. Le «sacrifice» ${ }^{105}$ de Montauran et de Marie est donc l'événement indispensable à cette construction qui ne peut être envisagée que si les deux parties versent un sang égal - la destruction totale de l'ancienne société va de pair avec le sacrifice des soldats de la Révolution.

Ainsi, malgré le drame annoncé par le titre du troisième chapitre - «Un jour sans lendemain»-Balzac développe des idées qu'on pourrait qualifier de tragiquement optimistes. À côté des royalistes «assez hardis, assez fanatiques, assez calculateurs» ${ }^{106}$, même si leur chef veut «du passé, l'avenir» ${ }^{107}$, Hulot et ses soldats sont tous présentés comme d'honnêtes républicains, «dévoués» et «nobles». Ils se battent héroïquement pour le «pays», pour un meilleur «avenir»:

Voilà un [Gérard] de mes hommes à grandes vues, se dit Mlle de Verneuil. Appuyés sur le présent qu'ils dominent, ils ruinent le passé, mais au profit de l'avenir... ${ }^{108}$.

D'ailleurs, bien que le mariage soit «sans lendemain», ses six heures de «bonheur» et de «volupté» sont une éternité:

Elle se retourna et contempla le marquis endormi [...] comme s'il se fût endormi au milieu d'un baiser [...] elle excitait si bien le désir en paraissant s'y refuser [...] Ils relevèrent ensemble leurs têtes charmantes, et se firent mutuellement un signe de reconnaissance plein des plaisirs qu'ils avaient goûtés ${ }^{109}$.

Invraisemblablement, à ce moment-là, de l'autre côté de ce «lit fatal» ${ }^{110}$, les soldats Bleus et leur chef restent «immobiles», attendant patiemment la fin de cette nuit de noces. Il est donc important pour l'auteur que le «mariage» soit consommé afin que l'union entre l'Ancien Régime et la République soit elle-même établie dans l'union des corps des deux époux.

Enfin, à la vue de ses bourreaux, installés par sa propre femme, le mari accepte le fait et n'a aucune hésitation avant de lui pardonner: «Va! je t'aime toujours» ${ }^{111}$. Immé-

tout bas Francine»; 1ère éd.: «C'est une sainte!... dit tout bas Francine» (p. 360).

101 «Et bien! Pierre, s'il lui [Marie] arrive le moindre malheur, si un seul cheveu de sa tête est arraché, nous nous serons vus ici pour la dernière fois et pour l'éternité, car je serai dans le paradis, moi! et toi, tu iras en enfer», op. cit., p. 997 (p. 120).

(102) Op. cit., p. 950 (p. 65).

(103) Op. cit., pp. 1007, 1014 (p. 136). Dans les versions corrigées, après que Montauran a dévoilé son identité non républicaine à Marie, celle-ci annonce que «la tragédie a bien promptement commencé». Bien que ce moment précis ne soit pas désigné de façon explicite en 1829, les mots «tragédie» et «drame» sont aussi employés.

(104) Op. cit., p. 1014 (p. 136).

(105) C'est le mot essentiel de Joseph de Maistre.

(106) Op. cit., p. 1046 (p. 170).

(107) Ibid. (p. 170).

(108) Op. cit., p. 1045 (p. 170).

(109) Op. cit., p. 1207. L'expression «plein de plaisirs» a remplacé une autre expression: «plein de gentillesse» dans la première version (pp. 362-363).

(110) Op. cit., p. 1207 (p. 362).

(111) Ibid. 1ère éd.: «Et bien!, je t'aime encore!...dit-il» (p. 363). 
diatement, grâce à cette générosité sans borne, le désarroi de Marie s'évapore - elle est comme rassurée et optimiste: «Tout n'est donc pas perdu [...] il y a de l'espoir» ${ }^{12}$, enfin réconciliée, corps et âme, avec le marquis. Plus rien ne peut troubler cet ultime bonheur. La même attitude se trouve chez le marquis, pour qui la guerre a disparu, devant le lit nuptial: «Eh! laissons-les, Marie, et reviens» ${ }^{113}$. Obligé d'aller affronter les Bleus, le chef royaliste n'a plus rien de conservateur.

Grâce à ce dénouement qui devrait être prophétique, la réconciliation des deux France peut être envisagée. La mort du marquis et de la «marquise» ${ }^{114}$ de Montauran n'est rien d'autre que la concrétisation de la vision positive de Balzac, fondée sur la croyance en la générosité de l'«Amour» ${ }^{15}$. Cet optimisme, la suite de l'œuvre de Balzac (La Cousine Bette, Les Paysans etc.), va le mettre à mal dans des drames qui n'auront rien à voir avec la «tragédie» que nous venons de lire.

CHIA-PING KAN

(112) Ibid. (p. 363).

(113) Ibid. (p. 363).

(114) Op. cit., p. 1208 (p. 362, p. 364).

(115) À la différence de la première version, Les Chouans ne se termine pas sur la mort de Montau- ran et de Marie, mais sur une scène quotidienne du marché avec Marche-à-terre et Francine en 1827. Les violents conflits entre les royalistes et les républicains n'auront été qu'un passage de l'Histoire, une Histoire définie comme succession de passages. 